\title{
Hydrothermal Convection in Moderately Thin Spherical Shells
}

\author{
Zhifeng Dai, ${ }^{1}$ Keke Zhang, ${ }^{1}$ Xinhao Liao, ${ }^{2}$ and Gerald Schubert ${ }^{3}$ \\ ${ }^{1}$ Department of Mathematical Sciences, University of Exeter, EX4 4QE, United Kingdom \\ ${ }^{2}$ Shanghai Astronomical Observatory, Chinese Academy of Sciences, Shanghai 200030, China \\ ${ }^{3}$ Department of Earth and Space Sciences and Institute of Geophysics and Planetary Physics, University of California,
} Los Angeles, California 90095-1567, USA

(Received 25 March 2008; published 8 July 2008)

\begin{abstract}
Hydrothermal convection of pore water with a temperature-dependent viscosity within a permeable, internally heated, moderately thin spherical shell is investigated by both a perturbation analysis and a direct numerical simulation. The analysis and simulation are mainly focused on a thin spherical shell in that convective instabilities are characterized by the spherical harmonic degree $l=6$ with a 13 -fold mathematical degeneracy. Four different three-dimensional analytical solutions of convection are derived by removing the degeneracy through the nonlinear effect. A direct numerical simulation of the nonlinear problem is also carried out, showing satisfactory agreement between the analytical solutions and the numerical simulations.
\end{abstract}

PACS numbers: 91.45.Fj

Introduction. - Convection of water through a sphericalshell porous medium with a central gravitational force and internal heating is likely to have occurred during the evolution of a number of solar system bodies. During the stage of early heating of carbonaceous chondrite parent bodies [1], ice at the center of the body will melt first and the released rock will sink to the center forming a rock core. It is likely that liquid water did not simply fill the porosity of the parent bodies but circulated in a convective manner similar to what is observed in geothermal systems in Earth's crust [2]. Hydrothermal convection through rocky spherical shells is also likely to have occurred or to be presently active in a number of outer planet icy satellites that have water-ice shells surrounding rocky cores, among which are Europa and Enceladus [3]. Understanding the flow structure of hydrothermal convection in a sphericalshell porous medium helps us elucidate the evolution of these celestial bodies. Moreover, since many astrophysical bodies are characterized by spherical-shell geometry and a spherically symmetric basic state, the mathematical problem classifying possible structures of nonlinear convection in the vicinity of the critical point, though this study is particularly concerned with hydrothermal convection in a spherical porous medium shell, is of relevance to many important problems in astrophysics.

Our theoretical knowledge and understanding of hydrothermal convection in a spherical-shell porous medium is, however, highly limited. This is primarily due to the fact that the convection problem with a spherically symmetric basic state and a spherically symmetric boundary condition is mathematically complicated, the linear stability analysis only determines the critical eigenfunction of the spherical harmonics $Y_{l}^{m}(\theta, \phi)$ of large degree $l$ for moderately thin spherical shells (e.g., [4]). Hence there exists a $(2 l+1)$ fold degeneracy, and a complete elimination of the degeneracy by nonlinearity proves to be a highly challenging task. Chossat [5] and Matthews [6] employed the group theoretical method in an abstract mathematical system to classify possible nonlinear solutions that may exist in the vicinity of the critical bifurcation point in an abstract mathematical system. In an important study, Busse [7] considered a weakly nonlinear problem in spherical geometry without reference to the physical detail of a convection system and with an assumption that all solutions of the problem possess symmetry with respect to a plane through the center of the sphere. Nonlinear analysis that derives an analytical solution for hydrothermal convection in a spherical-shell porous medium and its verification by a direct three-dimensional numerical simulation have not been attempted.

In this Letter, both the mathematical analysis and the corresponding numerical simulations of weakly nonlinear hydrothermal convection in moderately thin spherical shells of pore water with a temperature-dependent viscosity are carried out, showing satisfactory agreement between the analytical and numerical solutions.

The model and mathematical formulation. - We consider a spherical shell, with inner radius $r_{i}$ and outer radius $r_{0}$, of water-saturated permeable porous material heated by a uniformly distributed internal heat source which produces a linear radial temperature gradient $\partial T_{0} / \partial r=$ $-\beta r$, where $T_{0}$ is the conduction temperature, $\beta$ is a constant, and $r$ is the distance from the center of the spherical system. Water, with the thermal expansivity $\alpha$, fills all of the pore space with volumetric porosity $\Phi$. An appropriate scaling [2] of Darcy's law, the equation of continuity, and the heat equation leads to the dimensionless governing equations for hydrothermal convection

$$
\begin{gathered}
0=-\nabla P+R \mathbf{r} \Theta-\hat{\mu} \mathbf{u}, \\
0=\nabla \cdot \mathbf{u}, \\
\frac{\partial \Theta}{\partial t}+\mathbf{u} \cdot \nabla \Theta=\mathbf{r} \cdot \mathbf{u}+\nabla^{2} \Theta .
\end{gathered}
$$


Here $\mathbf{u}$ is the dimensionless Darcy velocity, $\Theta$ and $P$ denote the departures of temperature and pressure from their values in the basic static state, the Rayleigh number $R$ is defined as $R=\alpha \beta \gamma\left(r_{0}-r_{i}\right)^{4} / K \kappa$, where $K$ is inversely proportional to the permeability, $\kappa$ is proportional to the averaged thermal conductivity of the water and rock and $\gamma$ is related to gravity by $\mathbf{g}=-\gamma \mathbf{r}$, and $\hat{\mu}$ is the temperaturedependent viscosity (see also [8] which discussed the convection problem for the whole sphere). Equations (1)-(3) are subject to the boundary conditions at the inner radius $r_{i}$ and the outer radius $r_{0}$ of the shell

$$
\mathbf{r} \cdot \mathbf{u}=\Theta=0 \text {. }
$$

It is mathematically convenient to express the Darcy velocity $\mathbf{u}$ in terms of poloidal $(v)$ and toroidal $(w)$ components in the form

$$
\mathbf{u}=\nabla \times \nabla \times(\mathbf{r} v)+\nabla \times(\mathbf{r} w),
$$

which satisfies (2) automatically. The scalar equations for $v$ and $w$ can be obtained by substituting (5) into (1) and then applying the operators $\mathbf{r} \cdot \nabla \times$ and $\mathbf{r} \cdot \nabla \times \nabla \times$ onto the resulting equation, which yields the two equations

$$
\begin{gathered}
\mathbf{r} \cdot \nabla \times[\hat{\mu} \nabla \times(\mathbf{r} w)+\hat{\mu} \nabla \times \nabla \times(\mathbf{r} v)]=0, \\
R L^{2} \Theta-\mathbf{r} \cdot \nabla \times \nabla \times[\hat{\mu} \nabla \times(\mathbf{r} w)+\hat{\mu} \nabla \times \nabla \times(\mathbf{r} v)]=0,
\end{gathered}
$$

where $L^{2}$ denotes the differential operator representing the negative Laplacian on a unit sphere

$$
L^{2}=r^{2}\left(\frac{\partial^{2}}{\partial r^{2}}+\frac{2}{r} \frac{\partial}{\partial r}-\nabla^{2}\right)
$$

The heat equation (3) in terms of the poloidal flow $v$ becomes

$$
\frac{\partial \Theta}{\partial t}+\mathbf{u} \cdot \nabla \Theta=L^{2} v+\nabla^{2} \Theta .
$$

The boundary condition (4) can be then written as

$$
v=\Theta=0, \quad \text { at } \quad r=r_{i} \text { and } r_{0} .
$$

We first solve (6)-(8) subject to (9) analytically and determine the structure of hydrothermal convection which will then be verified by a direct numerical simulation.

Perturbation analysis and numerical simulations. - We first derive small amplitude solutions near the onset of convective instability of (6)-(8) satisfying (9) through a perturbation analysis. We expand $v, w, R$ and $\hat{\mu}$ in terms of small but nonzero amplitude $\epsilon$ of convection,

$$
\begin{gathered}
v=\epsilon v_{0}+\epsilon^{2} v_{1}+\ldots, \\
\Theta=\epsilon \Theta_{0}+\epsilon^{2} \Theta_{1}+\ldots
\end{gathered}
$$

$$
\begin{gathered}
R=R_{0}+\epsilon R_{1}+\epsilon^{2} R_{2} \ldots, \\
\hat{\mu}=\hat{\mu}_{0}(r)-\epsilon \hat{\mu}_{1}(r) \Theta_{0}-\epsilon^{2} \hat{\mu}_{1}(r) \Theta_{1}+\ldots,
\end{gathered}
$$

where we take $\hat{\mu}_{0}=\hat{\mu}_{1}=10^{q\left(r-r_{i}\right)^{2}}$ for water [9] (see also [8]) with $q=0.05$ used in our simulation. We also assume that the exchange of instability is valid in our nonlinear analysis. Substitution of (10)-(13) into (6)-(8) gives the leading-order equations describing the onset of instability

$$
R_{0} L^{2} \Theta_{0}+\hat{\mu}_{0}(r) L^{2} \nabla^{2} v_{0}+\frac{1}{r} \frac{\partial \hat{\mu}_{0}}{\partial r} L^{2}\left[\frac{\partial}{\partial r}\left(r v_{0}\right)\right]=0,
$$

$$
\frac{\partial \Theta_{0}}{\partial t}=L^{2} v_{0}+\nabla^{2} \Theta_{0},
$$

subject to the boundary conditions

$$
v_{0}=\Theta_{0}=0, \quad \text { at } \quad r=r_{i} \text { and } r_{0} .
$$

For moderately thin spherical shells, for example, at $r_{i} / r_{0}=0.6$ on which we shall focus, the general solution of the leading-order problem can be written as

$$
\begin{array}{r}
\boldsymbol{v}_{0}=\sum_{m=-6}^{m=6} Z_{m} P_{6}^{m}(\theta) e^{i m \varphi}\left[\sum_{n=1}^{N} c_{n} f_{l}\left(\xi_{n} r\right)\right], \\
\Theta_{0}=\sum_{m=-6}^{m=6} Z_{m} P_{6}^{m}(\theta) e^{i m \varphi}\left[\sum_{n=1}^{N} \frac{42 c_{n}}{\xi_{n}^{2}} f_{l}\left(\xi_{n} r\right)\right],
\end{array}
$$

with

$$
f_{l}\left(\xi_{n} r\right)=n_{l}\left(\xi_{n} r_{i}\right) j_{l}\left(\xi_{n} r\right)-j_{l}\left(\xi_{n} r_{i}\right) n_{l}\left(\xi_{n} r\right),
$$

where $j_{l}(z)$ and $n_{l}(z)$ denote the spherical Bessel functions of the first and second kinds for $l=6, \xi_{n}$ is determined by the equation $f_{6}\left(\xi_{n} r_{0}\right)=0, N$ is the truncation parameter usually taken as $N=O(10), R_{0}$ and $c_{n}$ can be determined by the linear stability analysis, and $P_{l}^{m}(\theta)$ is the associated Legendre function normalized such that

$$
\frac{1}{4 \pi} \int_{0}^{2 \pi} \int_{0}^{\pi}\left\{\left|P_{l}^{m}(\theta) e^{i m \phi}\right|^{2}\right\} \sin \theta d \theta d \phi=1 .
$$

Note that all coefficients $c_{n}$ in (17) and (18) can be determined by the linear stability analysis. It is important to notice that (i) the leading-order problem is not self-adjoint although its adjoint problem, the solution of which will be denoted by $v_{0}^{+}$and $\Theta_{0}^{+}$, can be derived in a similar way, and (ii) the values of complex coefficients $Z_{m}$ cannot be determined by the stability analysis. The complex coefficients $Z_{m}$ are determined by the nonlinear effect in connection with the $O\left(\epsilon^{2}\right)$ problem governed by

$$
\hat{\mu}_{0} L^{2} w_{1}=\mathbf{r} \cdot \nabla \times\left[\hat{\mu}_{1} \Theta_{0} \nabla \times \nabla \times\left(\mathbf{r} v_{0}\right)\right],
$$

$$
-R_{0} L^{2} \Theta_{1}-\hat{\mu}_{0} L^{2} \nabla^{2} v_{1}-\frac{1}{r} \frac{\partial \hat{\mu}_{0}}{\partial r} L^{2}\left[\frac{\partial}{\partial r}\left(r v_{1}\right)\right]=R_{1} L^{2} \Theta_{0}+\mathbf{r} \cdot \nabla \times \nabla \times\left[\hat{\mu}_{1} \Theta_{0} \nabla \times \nabla \times\left(\mathbf{r} v_{0}\right)\right],
$$




$$
L^{2} v_{1}+\nabla^{2} \Theta_{1}=\left[\nabla \times \nabla \times\left(\mathbf{r} v_{0}\right)\right] \cdot \nabla \Theta_{0},
$$

subject to the boundary conditions

$$
v_{1}=\Theta_{1}=0, \quad \text { at } \quad r=r_{i} \text { and } r_{0} .
$$

The solvability condition (19)-(21) can be written as

$$
R_{1} Z_{k} \int_{r_{i}}^{r_{o}} \frac{1}{\hat{\mu}_{0}} f_{l}^{+}(r) g_{l}(r) r^{2} d r=\frac{1}{4}\left(R_{0} I_{1}-I_{2}\right),
$$

where $l=6, k=0,1,2, \ldots, 6$, and

$$
\begin{aligned}
I_{1}= & \sum_{m=0}^{m=6} \sum_{q=0}^{q=6} Z_{m} Z_{q} \delta(m-k+q) \\
& \times \int_{0}^{\pi} P_{l}^{-k} P_{l}^{m} P_{l}^{q} \sin \theta d \theta\left[-\int_{r_{i}}^{r_{o}} \frac{d g_{l}^{+}}{d r} f_{l} g_{l} r d r\right. \\
& \left.+\int_{r_{i}}^{r_{0}} \frac{d g_{l}}{d r} f_{l} g_{l}^{+} r d r\right], \\
I_{2}= & \sum_{m=0}^{m=6} \sum_{q=0}^{q=6} Z_{m} Z_{q} \delta(m-k+q) \\
& \times \int_{0}^{\pi} P_{l}^{-k} P_{l}^{m} P_{l}^{q} \sin \theta d \theta\left[\int_{r_{i}}^{r_{0}} \hat{\mu}_{1} g_{l} \frac{d}{d r}\left(\frac{r f_{l}^{+}}{\hat{\mu}_{0}}\right)\right. \\
& \left.\times \frac{d\left(r f_{l}\right)}{d r} d r+l(l+1) \int_{r_{i}}^{r_{0}} \hat{\mu}_{1} g_{l}\left(\frac{f_{l}^{+}}{\hat{\mu}_{0}}\right) f_{l} d r\right]
\end{aligned}
$$

where $f_{l}^{+}$and $g_{l}^{+}$are the adjoint solution of the leadingorder problem, $\delta(n=0)=1$ and $\delta(n \neq 0)=0$. The nonlinear equation (23) can be solved for determining the structure of the hydrothermal convection. After an extensive search, four different solutions of three-dimensional convection are derived. The first analytical solution contains only one associated Legendre function given by

$$
\begin{gathered}
v=\left[\frac{\sqrt{13}(R-10.43)}{0.10487}\right] P_{6}^{0}(\theta)\left[\sum_{n=1}^{N} c_{n} f_{l}\left(\xi_{n} r\right)\right], \\
\Theta=\left[\frac{\sqrt{13}(R-10.43)}{0.10487}\right] P_{6}^{0}(\theta)\left[\sum_{n=1}^{N} \frac{42 c_{n}}{\xi_{n}^{2}} f_{l}\left(\xi_{n} r\right)\right] .
\end{gathered}
$$

The second convection solution comprises two associated Legendre functions $P_{6}^{0}(\theta)$ and $P_{6}^{6}(\theta)$

$$
\begin{aligned}
v=[ & \left.\frac{\sqrt{13}(R-10.43)}{0.067632}\right]\left[\sum_{n=1}^{N} c_{n} f_{l}\left(\xi_{n} r\right)\right] \\
\times & {\left[0.056302 P_{6}^{0}(\theta)-\frac{0.810093}{720 \sqrt{231}} P_{6}^{6}(\theta) \sin (6 \phi)\right], } \\
\Theta= & {\left[\frac{\sqrt{13}(R-10.43)}{0.067632}\right]\left[\sum_{n=1}^{N} \frac{42 c_{n}}{\xi_{n}^{2}} f_{l}\left(\xi_{n} r\right)\right] } \\
& \times\left[0.056302 P_{6}^{0}(\theta)-\frac{0.810093}{720 \sqrt{231}} P_{6}^{6}(\theta) \sin (6 \phi)\right] .
\end{aligned}
$$

The third solution comprises two associated Legendre functions $P_{6}^{0}(\theta)$ and $P_{6}^{5}(\theta)$

$$
\begin{aligned}
v= & {\left[\frac{\sqrt{13}(R-10.43)}{0.225439}\right]\left[\sum_{n=1}^{N} c_{n} f_{l}\left(\xi_{n} r\right)\right] } \\
& \times\left[0.589302 P_{6}^{0}(\theta)-\frac{0.62361}{360 \sqrt{77}} P_{6}^{5}(\theta) \sin (5 \phi)\right], \\
\Theta= & {\left[\frac{\sqrt{13}(R-10.43)}{0.225439}\right]\left[\sum_{n=1}^{N} \frac{42 c_{n}}{\xi_{n}^{2}} f_{l}\left(\xi_{n} r\right)\right] } \\
& \times\left[0.589302 P_{6}^{0}(\theta)-\frac{0.62361}{360 \sqrt{77}} P_{6}^{5}(\theta) \sin (5 \phi)\right] .
\end{aligned}
$$

The fourth solution also contains two associated Legendre
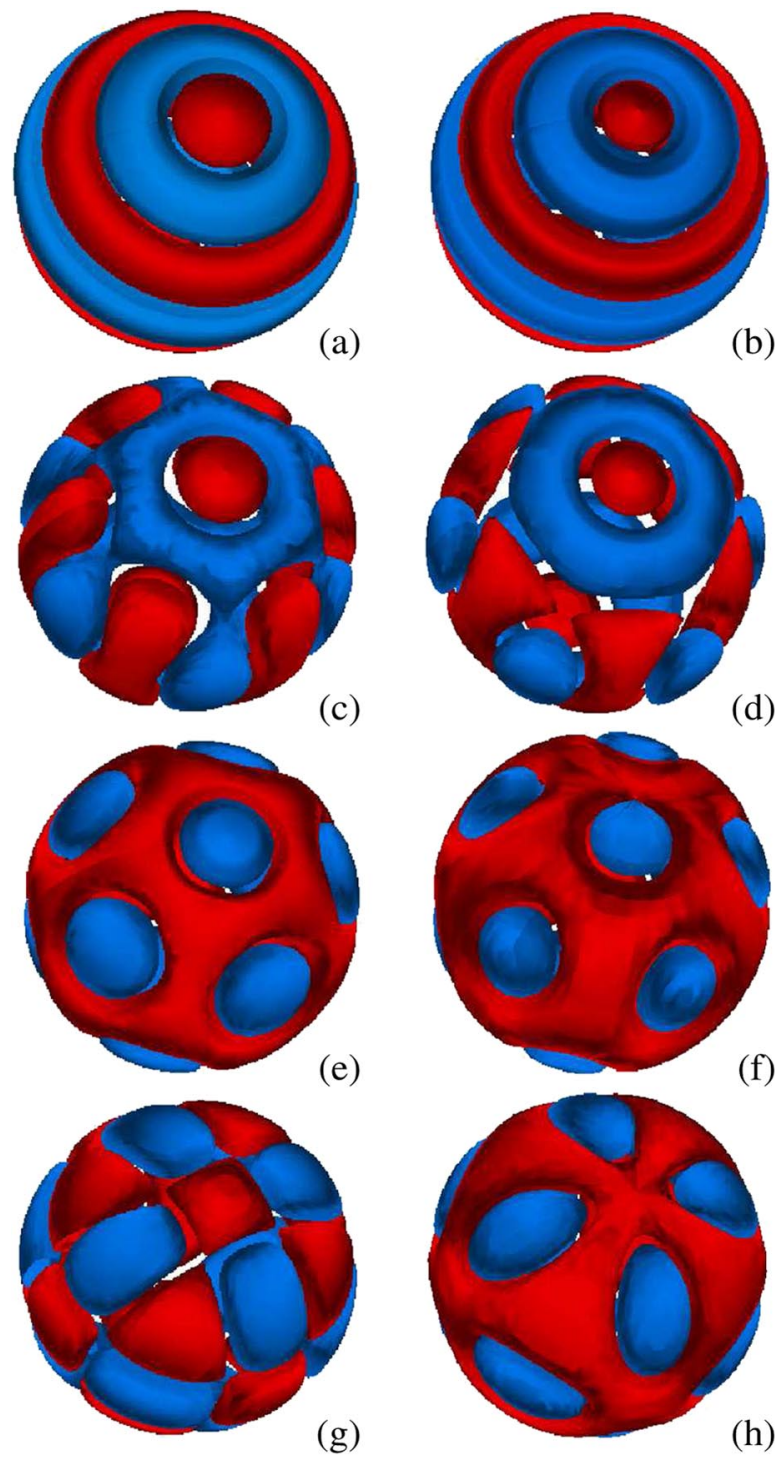

FIG. 1 (color online). Isosurfaces of the temperature of convection for $r_{i} / r_{0}=0.6$. Red contours indicate $\Theta>0$ and blue contours correspond to $\Theta<0$. The left panels (a),(c),(e),(g) show analytical solutions while the right column shows the corresponding numerical simulations. 


$$
\begin{aligned}
& \text { functions } P_{6}^{0}(\theta) \text { and } P_{6}^{4}(\theta) \\
& \begin{aligned}
v= & \left.\frac{\sqrt{13}(R-10.43)}{0.019774}\right)\left[\sum_{n=1}^{N} c_{n} f_{l}\left(\xi_{n} r\right)\right] \\
& \times\left[0.471405 P_{6}^{0}(\theta)-\frac{0.881917}{120 \sqrt{14}} P_{6}^{4}(\theta) \sin (4 \phi)\right], \\
\Theta= & \left(\frac{\sqrt{13}(R-10.43)}{0.019774}\right)\left[\sum_{n=1}^{N} \frac{42 c_{n}}{\xi_{n}^{2}} f_{l}\left(\xi_{n} r\right)\right] \\
& \times\left[0.471405 P_{6}^{0}(\theta)-\frac{0.881917}{120 \sqrt{14}} P_{6}^{4}(\theta) \sin (4 \phi)\right] .
\end{aligned}
\end{aligned}
$$

The profiles of four different analytical solutions are shown in the left column of Fig. 1.

A huge effort is made to simulate directly the nonlinear equations (1) and (3) subject to the boundary condition (4) using a three-dimensional finite difference scheme based on uniform grids in the azimuthal direction but nonuniform grids in the latitudinal and radial directions. The particular numerical scheme, discussed in [10] which is only concerned with convection of constant viscosity, produces a system of finite difference equations in the tridiagonal form which can be readily solved by a domain decomposition method. By fixing the Rayleigh number $R$ at a value that is slightly above the critical Rayleigh number $\left(R-R_{0}\right)=$ 0.57 , where $R_{0}=10.43$ for the case $r_{i} / r_{0}=0.6$, and by changing the initial conditions, we are also able to obtain four different numerical solutions of the nonlinear hydrothermal convection all of which are stationary. The profiles of the four numerical solutions are depicted in the right column of Fig. 1. A comparison of the left and right columns shows satisfactory agreement between the analytical solutions given by (24)-(31) and the nonlinear simulation of a direct numerical integration of equations (1)-(3). Some noticeable differences should be anticipated since our numerical simulations are performed at a finite supercritical Rayleigh number $\left(R-R_{0}\right)=0.57$. For example, the excitation of other spherical harmonics and the resulting modification at a moderate supercritical Rayleigh number used in simulations may explain the differences between Figs. 1(g) and 1(h). A direct threedimensional numerical integration of the nonlinear convection at a very small supercritical Rayleigh number is extremely computationally expensive.

Concluding remarks. - A linear stability analysis for hydrothermal convection in a moderately thin shell only determines the critical Rayleigh number $R_{0}$ at which the basic motionless state becomes unstable with respect to infinitesimal disturbances characterized by the spherical harmonics of large degree $l$. For $r_{i} / r_{0}=0.6$, there exists a 13-fold degeneracy. In this Letter, we present the first mathematical analysis for a realistic physical problem of spherical convection that eliminates the degeneracy by nonlinearity and determines the structure of convective flows. It is also the first time that satisfactory agreement between the analytical solutions and direct numerical simulations for hydrothermal spherical convection is achieved.

It is important to point out that, in addition to the four simplest solutions discussed, we have also found many other analytical solutions which usually appear to be much more complicated, comprising more than two Legendre functions in the expression of the solutions. However, we are able to show that the three-dimensional structures of the additional analytical solutions are exactly the same as the four solutions after performing an appropriate rotational transformation. This is because the position of a pole or an equator in the convection system is arbitrary as a consequence of the spherically symmetric basic state and boundary condition. Our mathematical analysis and numerical simulation suggest that there are a variety of different patterns of water circulation through the porous spherical shells in planetesimals and icy satellites.

K.Z. is supported by UK NERC and PPARC grants, X. L. is supported by NSFC grant No. 10633030 and CAS grant No. KJCX2-YW-T13 and G.S. is supported by NASA Planetary Geology and Geophysics grant No. NNG06GG70G. The numerical computation is supported by SSC.

[1] R. E. Grimm and H. Y. McSween, Icarus 82, 244 (1989).

[2] E. D. Young, K. Zhang, and G. Schubert, Earth and Planet Science Lett. 213, 249 (2003).

[3] G. Schubert, J. D. Anderson, B. J. Travis, and J. Palguta, Icarus 188, 345 (2007).

[4] L. Li, P. Zhang, X. Liao, and K. Zhang, Phys. Rev. E 71, 016301 (2005).

[5] P. Chossat SIAM J. Appl. Math. 37, 624 (1979).

[6] P. C. Matthews, Phys. Rev. E 67, 036206 (2003).

[7] F. H. Busse, J. Fluid Mech. 72, 67 (1975).

[8] K. Zhang, X. Liao, and G. Schubert, Phys. Fluids 17, 086602 (2005).

[9] J. V. Sengers and J. T. R. Watson, J. Phys. Chem. Ref. Data 15, 1291 (1986).

[10] Z. Dai, K. Zhang, G. Schubert, and X. Liao, Phys. Fluids 20, 026601 (2008). 